\title{
A Phenomenological Description of Primary Creep in Class M Materials
}

\author{
S. V. Raj and A. D. Freed \\ NASA Glenn Research Center at Lewis Fields, \\ 21000 Brookpark Road, Cleveland, OH 44135
}

\begin{abstract}
Observations of creep microstructures in the primary creep region in class $M$ materials show a remarkable similarity with those formed in the exponential creep regime. As a result, it is proposed that the constitutive creep law for normal primary creep is similar to that for the exponential creep regime. A phenomenological description is discussed to rationalize these microstructural observations in terms of a normalized strain rate vs. stress plot. The implications of this plot in describing different testing procedures, steady-state flow, and on the observed deviations from the universal creep law are discussed. The plot is also extended to explain the observed similarities in the transient creep behavior in pre-strained materials and in stress change experiments.
\end{abstract}




\section{Discrepancies between the universal creep law and experimental observations}

A considerable amount of experimental data exists on the deformation behavior of materials. As a result, it is well established that the deformation of materials can be expressed in terms of a generalized rate equation given by [1]

$$
\dot{\varepsilon}=\mathrm{f}\left(\sigma, \mathrm{T}, \mathrm{S}_{\mathrm{i}}, \mathrm{P}_{\mathrm{j}}\right)
$$

called the kinetics equation and a generalized microstructure evolution equation describing the rate of change of the microstructure during deformation

$$
\dot{S}_{i}=\mathrm{g}\left(\sigma, \mathrm{T}, S_{\mathrm{i}}, \mathrm{P}_{\mathrm{j}}\right)
$$

known as the internal state equation, where $\dot{\varepsilon}$ is the strain rate, $\sigma$ is the applied stress, T is the absolute temperature, $\mathrm{S}_{\mathrm{i}}$ is the ith microstructural parameter and $\mathrm{P}_{\mathrm{j}}$ is the $\mathrm{j}$ th material property. Equations (1) and (2) are fairly general and they are considered to be applicable to both low and high temperature deformation under either cyclic or monotonic stress conditions. An examination of eqns. (1) and (2) reveals that they are coupled through the microstructural parameters. Thus, the strain rate is influenced by the evolution of $S_{i}$ during creep.

Specifically, the secondary creep rate at intermediate values of normalized stress is given by a power-law relation

$$
\dot{\varepsilon}=\mathrm{A}\left(\frac{\sigma}{G}\right)^{\mathrm{n}} \exp \left(-\frac{Q_{C}}{R T}\right)
$$

where $A$ and $n$ are constants, $G$ is the shear modulus, $Q_{C}$ is the activation energy for creep and $\mathrm{R}$ is the universal gas constant. Most creep theories predict that $\mathrm{n}=3$ for Metal type or class $\mathrm{M}$ creep behavior when dislocation climb is the rate-controlling mechanism [2]. 
Although the semi-empirical power-law relation eqn. (3) with $n>3$ can often describe the stress dependence of the secondary creep rate fairly adequately in a large number of cases, it is often assumed that the governing creep mechanism is dislocation climb with a constant activation energy approximately equal to that for lattice self-diffusion, $\mathrm{Q}_{1}$. This assumption is generally valid since there is a considerable amount of experimental data which demonstrate that $\mathrm{Q}_{c} \approx \mathrm{Q}_{1}[10,11]$. However, this constant value of activation energy is not always observed even when $n \approx 4-5$, where $Q_{c}$ has been observed to be stress dependent (Fig. 1(a) and (b)) $[6,9,12,13]$. It may be noted that non-diffusional deformation models can rationalize this stress dependence. Additionally, the measured activation volume for creep, $\mathrm{V}^{*}$, is typically $5 \times 10^{2}-10^{4} \mathrm{~b}^{3}$, where $\mathrm{b}$ is the Burgers vector. These values are much larger than the theoretical value of $b^{3}$ if the contribution to the observed creep rate was only from dislocation climb.

\section{Microstructural similarities between the exponential and primary creep regions}

Microstructural studies have been conducted on $\mathrm{NaCl}$ single crystals $[14,15,16]$, where the substructures formed in both the power-law and the exponential creep were characterized in a systematic manner over a large range of stresses and temperatures. Although microstructural studies on other materials have been limited to a narrow range of stresses and temperature, it is interesting to note that the microstructural features observed in $\mathrm{NaCl}$ single crystals, such as cells and elongated and equiaxed subgrains, have also been observed in these materials [9]. Based on these similarities in the substructural features, it appears reasonable to assume that the power-law to exponential creep transitional microstructures in $\mathrm{NaCl}$ single crystals also generally pertain to other materials.

Detailed microstructural observations conducted on $\mathrm{NaCl}$ single crystals reveal that the creep substructure is fairly complex consisting of a mixture of cells, dislocations and subgrains. Although it was initially suggested that there is a sudden break down in the subgrain microstructure at the point of transition from power-law to exponential creep 
boundary width, $\mathrm{L}^{\mathrm{h}}$, to the diameter of the cell interior, $\mathrm{L}^{\mathrm{s}}$, expressed as a function normalized stress, $\sigma / \mathrm{G}$, show that $\mathrm{L}^{\mathrm{h}} / \mathrm{L}^{\mathrm{s}}$ tends towards a constant value of about 0.2 as the microstructure approaches a steady-state configuration even in the exponential creep region (Fig. 3) [9,21]. Figure 3 also reveals that large deviations from this value occur in the exponential creep region, especially at lower values of creep strain and at lower temperatures. Similarly, microstructural observations have revealed that $L^{h} / L^{s}$ tends towards a value of 0.2 with increasing strain and temperature [9]. For example, Fig. 4 shows the variation of $L^{h} / L^{s}$ with $\varepsilon$. Using a mathematical fit similar to that used in the viscoplasticity literature [22], it was proposed that

$$
\mathrm{X}=\frac{L^{h}}{L_{s}}=\left(\frac{A_{2}}{B_{2}}\right)+\left[\mathrm{X}_{0}-\left(\frac{A_{2}}{B_{2}}\right)\right] \exp \left(-\mathrm{B}_{2} \varepsilon\right)
$$

where $A_{2}$ and $B_{2}$ are constants and $X_{0}$ is the initial value of $X$ [9]. The first and second terms in the parenthesis represent hardening and softening components, respectively. It should be noted that experimentally $X$ approaches a steady-state value of 0.2 as $\varepsilon$ tends towards infinity, and therefore, $\mathrm{A}_{2} / \mathrm{B}_{2} \approx 0.2$.

\section{Constitutive law for primary creep}

The apparent similarity in the microstructures observed in the exponential creep regime and in the primary transient stages of a deforming class $M$ material leads to an important question: Does the constitutive creep law for primary creep exhibit an exponential stress dependence? In an early study, Raymond et al. [23] demonstrated by conducting a series of stress decrease tests in the primary creep region during creep of cold-rolled aluminum that the initial creep rates measured at the new reduced values of stress exhibit an exponential stress dependence. Similarly, more recent interpretations of "constant structure" creep data obtained from stress drop tests suggest that these results are best described in terms of an exponential law rather than power-law equation $[24,25,26]$. The exponential law commonly used to fit these experimental data is that employed to describe obstacle-controlled dislocation glide [1,27] 
these contributions are significant, then Fig. 5(b) suggests that $n>3$; values of $n \approx 3$ are attributed to the fact that contributions from the non-diffusional creep mechanisms are negligible.

If dislocation climb is the sole creep mechanism, then $\mathrm{V}^{*} \approx \mathrm{b}^{3}$, where $\mathrm{b}$ is the Burgers vector $[28,29]$. However, in actuality, the observed values of $\mathrm{V}^{*}$ lie between $5 \mathrm{x}$ $10^{2}-10^{4} b^{3}$ for many class $M$ materials which exhibit $n>3[30,31]$, and they are similar to values reported for obstacle-controlled glide $[28,29]$. These experimental values of $\mathrm{V}^{*}$ suggest that non-diffusional creep mechanisms contribute significantly to the overall creep rate. We note that these contributions from non-diffusional creep mechanisms are also incorporated in the Nix-Gibeling two-phase creep model [32,33].

Figure 5(b) is consistent with the exponential eqns. (4) and (5), where $\mathrm{B}$ (or $\mathrm{V}^{*}$ in eqn. (5)) is the internal state variable of evolution if it is inversely proportional to the obstacle strength. This fact becomes evident when eqns. (4), (5) and (6) are compared with each other. As stated earlier, eqns. (5) and (6) are identical when $\dot{\varepsilon}_{0}=A_{1}, Q_{c}=\Delta F$ and $\sigma=Q_{c} / V^{*}$, so that $B=Q_{c} G / R T \sigma$ in eqn. (4). In other words, for this specific case, the structure parameters, $\mathrm{S}_{\mathrm{l}}$, shown in Fig. 5(b) depend on $\sigma$. For example, at a constant value of $\sigma / \mathrm{G}$, as $\mathrm{B}$ (or $\mathrm{V}^{*}$ ) decreases with increasing $\sigma$, the normalized creep rate will decrease as the microstructure approaches a steady-state value (i.e. $S_{6}>S_{5}>S_{4}>S_{3}$ in Fig. 5).

If the line with a slope $\mathrm{n}$ is assumed to represent the point where the material exhibits a quasi-steady-state behavior, then Fig. 5(b) can be used to draw some inferences on material behavior. First, an extension of this line to values of $\sigma / \mathrm{G}>(\sigma / \mathrm{G})_{\mathrm{PLB}}$ into the exponential creep region suggests that this regime is a transient state that is bounded on one side by quasi-steady-state behavior. This tendency is confirmed by the quantitative microstructural data shown in Fig. 3. Additionally, it is noted that steady-state behavior is often observed during hot-deformation when $\varepsilon>1$, despite the fact that the hot working strain rates are more than $10^{4}$ higher than the creep rates measured in a typical laboratory 
type of transient can be observed in a class $M$ material when it has been substantially predeformed [14]. However, if the material overshoots the quasi-steady-state line, sigmoidal creep is expected to occur as the material tries to get back to this line.

The expected initial creep transient following stress change tests conducted in the primary creep region can be rationalized easily using Fig. 6(b). On changing the stress from one level to another, the initial microstructural path is along one of the $S_{1}$ curves. For example, if the microstructural state corresponds to the point $\mathrm{X}$ at a normalized stress, $(\sigma / \mathrm{G})$, prior to a stress change, then on increasing the normalized stress to $(\sigma / \mathrm{G})_{\mathrm{H}}$, the initial microstructural state at the new stress level will correspond to $Y$ in Fig. 6(b). Likewise, a decrease in the normalized stress from $(\sigma / \mathrm{G})$ to $(\sigma / \mathrm{G})_{\mathrm{L}}$, leads to initial microstructural state being at $Z$ at the reduced stress. Since the new initial microstructural states, $\mathrm{Y}$ and $\mathrm{Z}$, correspond to positions above the quasi-steady-state line in the $\dot{\varepsilon} \exp \left(\frac{Q_{C}}{R T}\right)-\sigma / G$ space, Fig. $6($ b) suggests that normal creep transients will be observed after the stress change. It is important to note that the material is still in the primary creep region before the stress change is conducted. Despite the fact that a considerable amount of stress reduction data exists, most of these studies have been conducted after large amounts of strain after true or near steady-state creep conditions were established. Therefore, only limited observations have been made of strain transients in the primary creep region. However, Raymond et al. [23] reported the observation of normal creep transients after stress reductions were conducted in the early stages of primary creep.

If a large stress reduction occurs in the primary creep region, such as that shown in Fig, 6(a), or the stress reduction occurs in the secondary region so that the point $\mathrm{Z}$ corresponds to a microstructural state below the quasi-steady-state line, then an inverse creep transient is expected to be observed after the stress reduction. For the latter case, there is a large body of data which confirm that inverse creep transients are indeed

\footnotetext{
${ }^{2}$ Although not specifically discussed in this paper, elastic and anelastic or negative creep are often observed after a stress reduction. The transient creep discussed in this paper specifically refers to the response of the material after elastic and anelastic strain has been recovered.
} 


\section{REFERENCES}

1. H. J. Frost and M. F. Ashby, Deformation-Mechanism Maps: The Plasticity and Creep of Metals and Ceramics, Pergamon, Oxford (1982).

2. J. Weertman, Rate Processes in Plastic Deformation of Materials (edited by J. C. M. $\mathrm{Li}$ and A. K. Mukherjee), American Society for Metals, Materials Park, OH, 315 (1975).

3. J. E. Bird, A. K. Mukherjee and J. E. Dorn, Quantitative Relation Between HighTemperature Creep Behavior and Structure (edited by D. G. Brandon and A. Rosen), Israel Universities Press, Jerusalem, 1965, pp. 255-342.

4. S. V. Raj, Scripta Metall. 20 (1986) 1333.

5. D. Caillard and J. L. Martin, Rev. Phys. Appl. 22 (1987) 169.

6. S. V. Raj and T. G. Langdon, Acta Metall. 37 (1989) 843.

7. W. D. Nix and B. Ilschner, Strength of Metals and Alloys (ICSMA 5) (edited by P. Haasen, V. Gerold and G. Kostorz), vol. 3, Pergamon Press, Oxford, 1980, pp. 15031530 .

8. S. V. Raj and T. G. Langdon, Acta Metall. 39 (1991) 1823.

9. S. V. Raj, I. S. Iskovitz and A. D. Freed, Unified Constitutive Laws of Plastic Deformation (edited by A. S. Krausz and K. Krausz), Academic Press, Inc., San Diego, CA, 1996, pp. 343-439.

10. O. D. Sherby and P. M. Burke, Prog. Mater. Sci. 13 91967) 325.

11. O. D. Sherby and J. Weertman, Acta Met. 27 (1979) 387.

12. S. V. Raj and G. Pharr, unpublished research, Rice University, Houston, Texas (1986).

13. M. Pahutová, J. Cadek and P. Ryš, Phil. Mag. 23 (1971) 509.

14. S. V. Raj, G. M. Pharr and J. D. Whittenberger, Mater. Sci. Eng. A 113 (1989) 161.

15. S. V. Raj and G. M. Pharr, Mater. Sci. Eng. A 122 (1989) 233.

16. S. V. Raj and G. M. Pharr, Amer. Ceram. Soc. 75 (1992) 347.

17. G. M. Pharr, Scripta Metall. 15 (1981) 717.

18. S. Takeuchi and A. S. Argon, J. Mater. Sci. 11 (1976) 1542.

19. A. H. Clauer, B. A. Wilcox and J. P. Hirth, Acta Metall. 18 (1970) 381. 


\section{Table of Figures}

Figure 1. Comparison of the stress dependence of the experimental activation energy, $Q_{c}$, for creep of (a) $\mathrm{Cu}[6]$ and (b) $\mathrm{NaCl}$ single crystals $[9,12]$ with that predicted for obstaclecontrolled glide, $\mathrm{Q}_{\mathrm{g}}$.

Figure 2. Creep substructures formed in the exponential creep regime in $\mathrm{NaCl}$ single crystals deformed at $473 \mathrm{~K}$ under a normalized stress of $7.5 \times 10^{-4}$ at creep strains of (a) 0.10 , (b) 0.25 and (c) $0.50[9,12]$.

Figure 3. Stress dependence of the ratio of cell boundary width, $\mathrm{L}^{\mathrm{h}}$, to the diameter of the cell interior, $\mathrm{L}^{\mathrm{s}}$, for $\mathrm{NaCl}$ single crystals deformed in the power-law and exponential creep regimes [21]; $(\sigma / \mathrm{G})_{\text {PLB }}$ is the observed power-law breakdown criterion. The data tend towards a steady-state value of $\mathrm{L}^{\mathrm{h}} / \mathrm{L}^{\mathrm{s}} \approx 0.2$.

Figure 4. Strain dependence of the ratio of cell boundary width, $\mathrm{L}^{\mathrm{h}}$, to the diameter of the cell interior, $\mathrm{L}^{\mathrm{s}}$, for $\mathrm{NaCl}$ single crystals deformed in the exponential creep regimes [9]; $\mathrm{T}=473 \mathrm{~K}$ and $\sigma=10 \mathrm{MPa}$.

Figure 5. Schematic plots demonstrating that the transient primary creep stages, A, B, C and $D$ of the constant stress creep curve shown in (a) correspond to the structuredependent (i.e. $S_{I}$ ) exponential curves in the normalized creep rate-normalized stress plot shown in (b); the deformation path followed in constant strain rate experiment is also shown in (b). The broken line with a slope of $n=3$ represents the natural creep law while the solid line with slope, $n$, corresponds to the experimentally observed result.

Figure 6. Modified plots of Fig. 5 used for qualitatively rationalizing the nature of transient creep in class $M$ materials. (a) A specimen deformed at a normalized stress, $(\sigma / \mathrm{G})_{1}$, is subsequently crept at a new value of normalized stress $(\sigma / \mathrm{G})_{2}$; the microstructural state at $\mathrm{E}$ is below the quasi-steady-state line of slope $\mathrm{n}$ and an inverse 


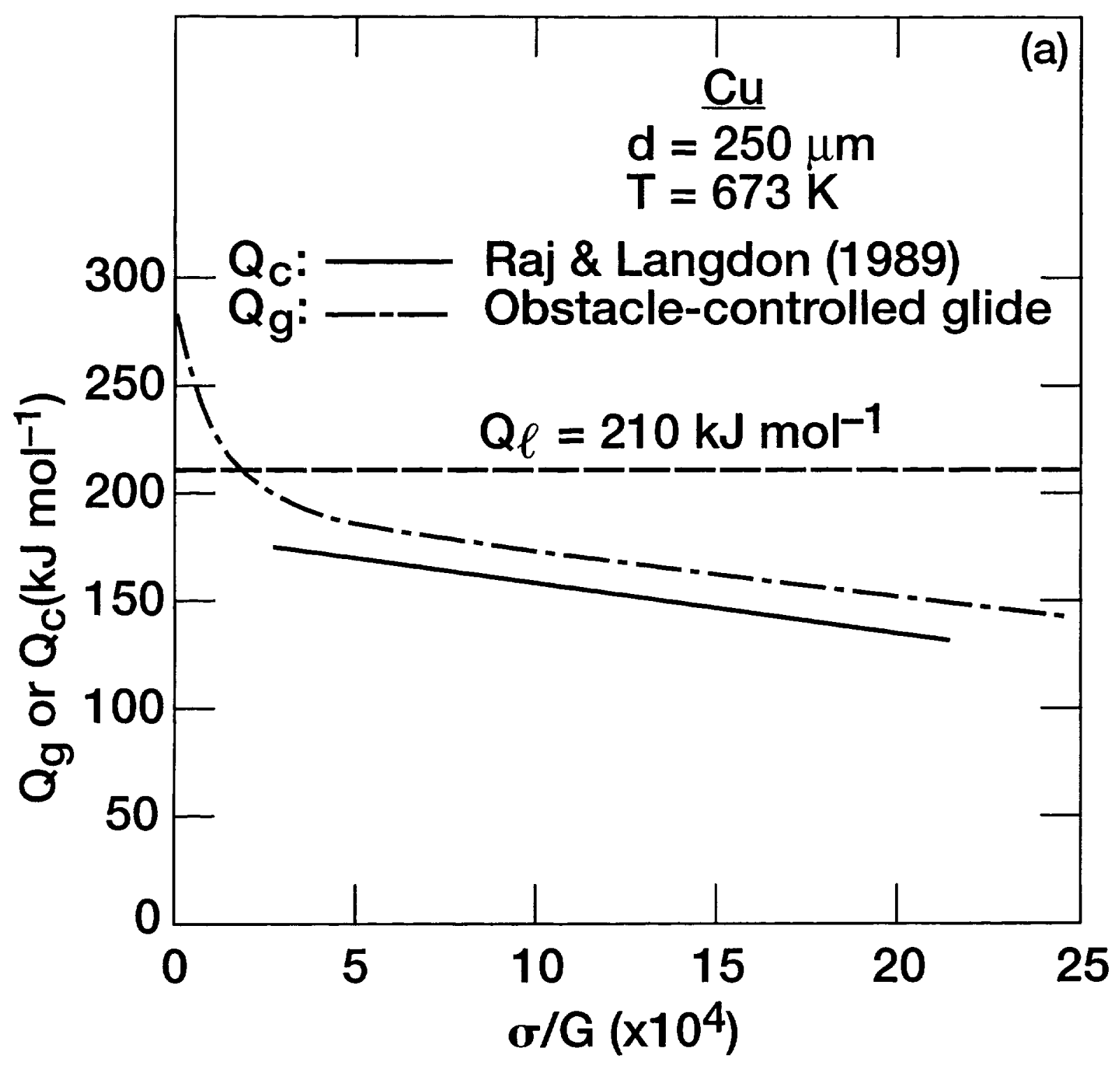

Fig 1a 


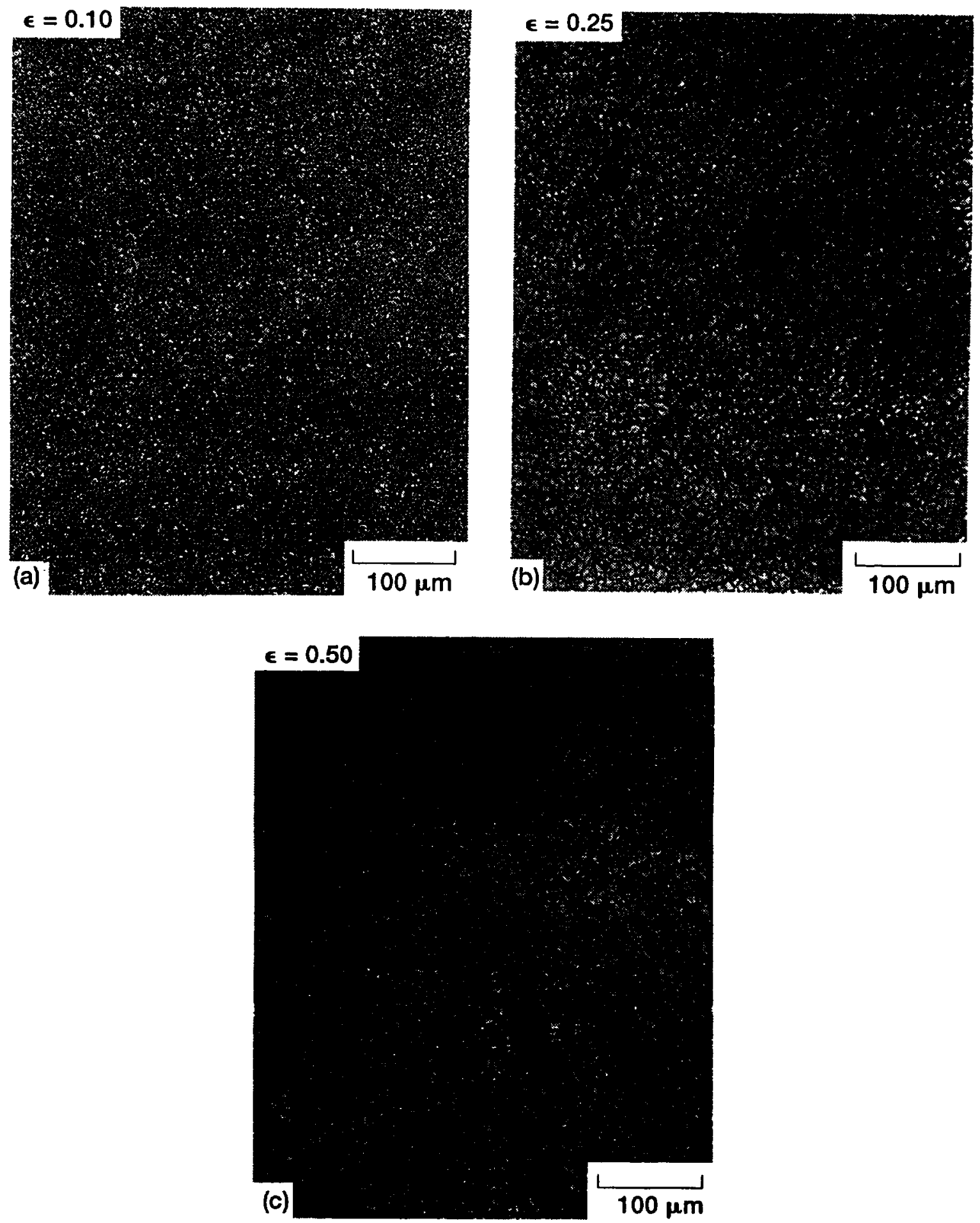




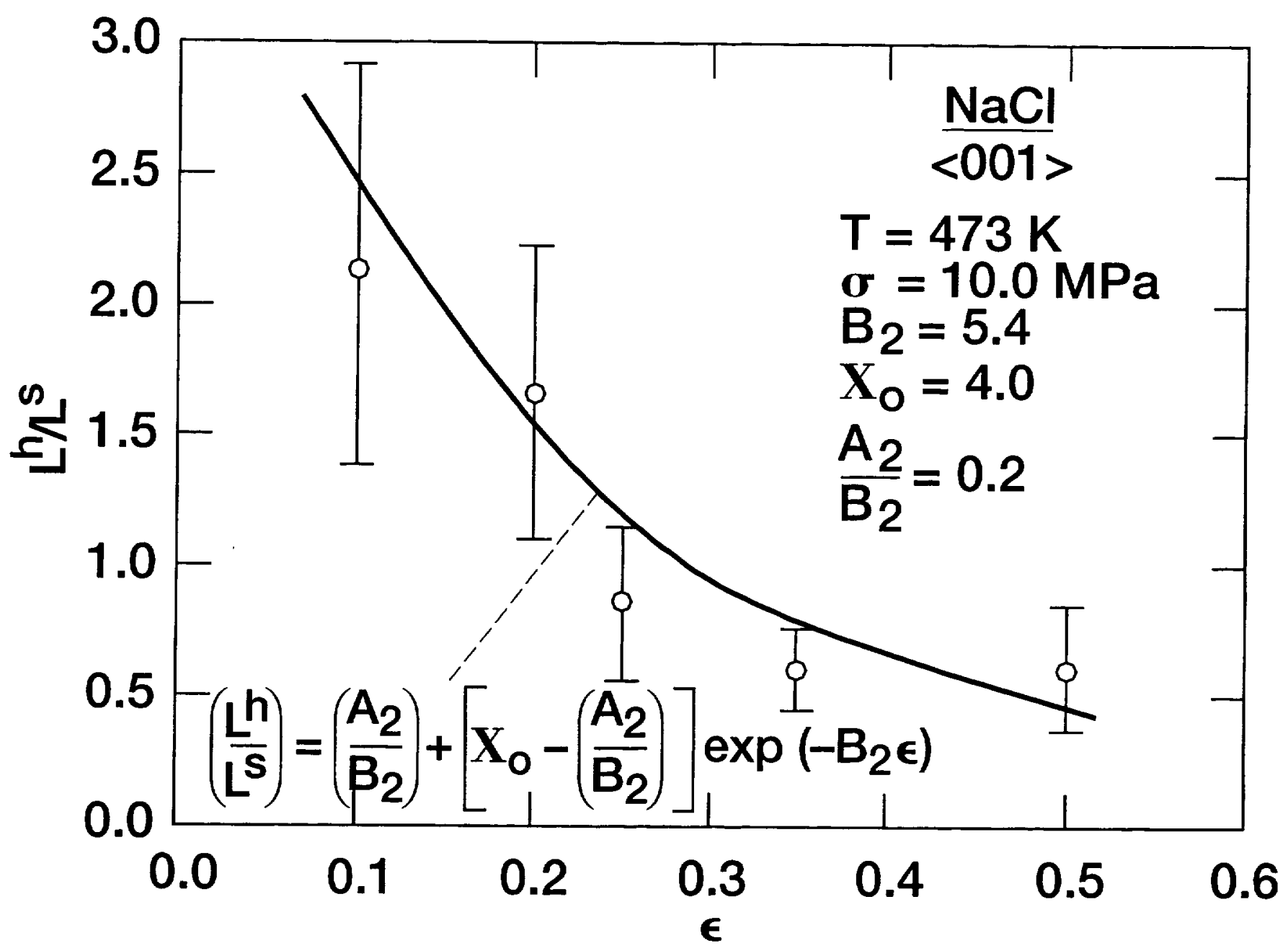

Fig 4 


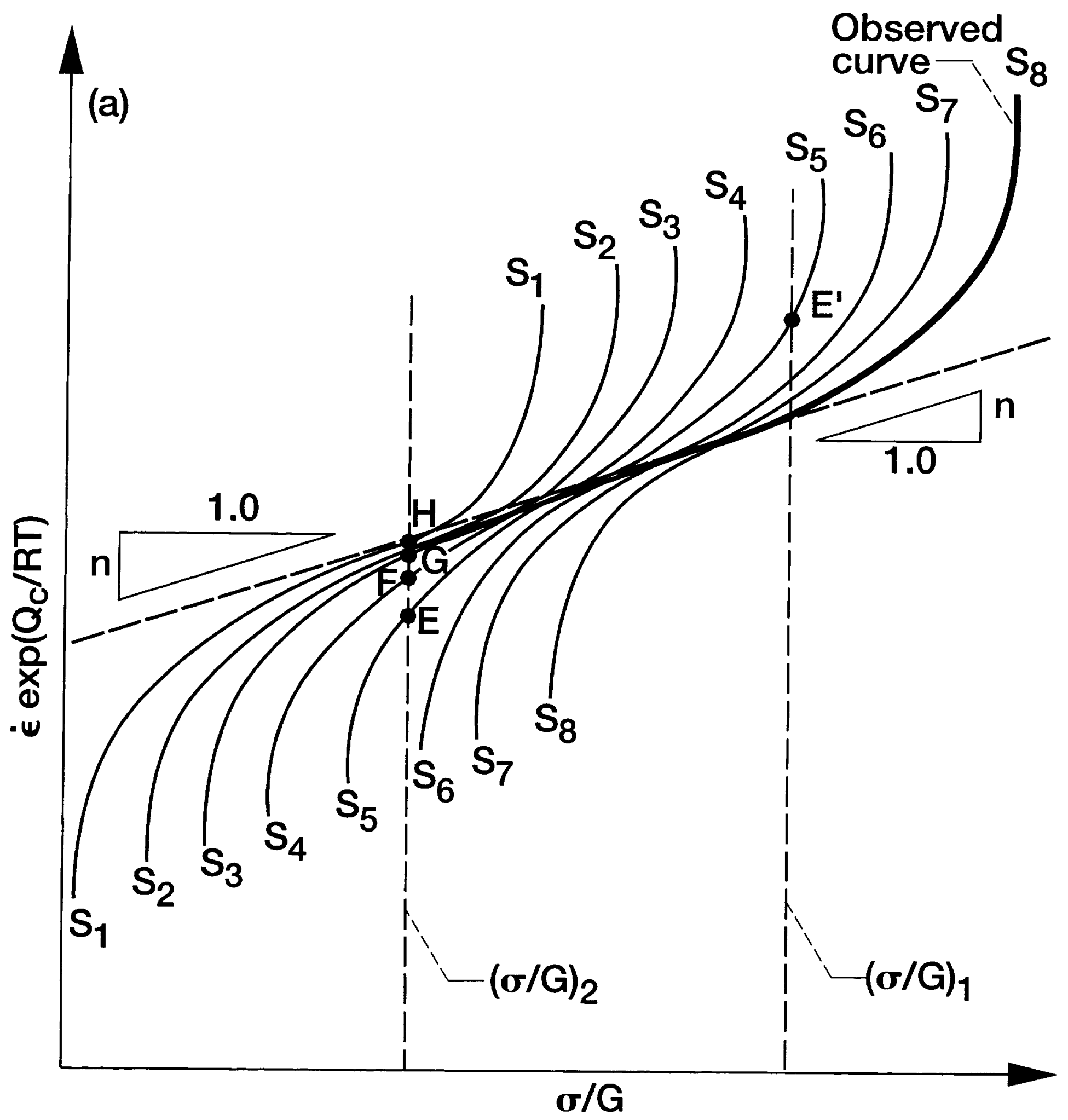

Fig $6 a$ 